\title{
Fatores associados ao desenvolvimento de ansiedade e depressão em estudantes de Odontologia
}

Clea Adas Saliba Garbin*; Luis Felipe Pupim dos Santos**; Artênio José Saliba Garbin***; Artênio José Ísper Garbin****; Tânia Adas Saliba****; Orlando Saliba*

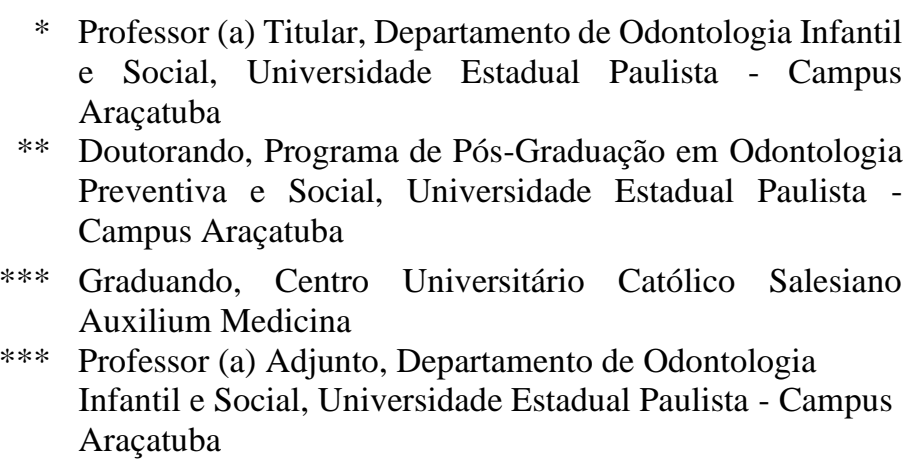

Recebido: 30/04/2020. Aprovado: 12/01/2021.

\begin{abstract}
RESUMO
O objetivo desta pesquisa foi avaliar os estudantes de uma faculdade pública de Odontologia localizada no Brasil, no que diz respeito à ansiedade e depressão, relacionando os escores de cada transtorno com as características pessoais, acadêmicas, e socioeconômicas dos discentes. Trata-se de um estudo transversal, no qual participaram 423 estudantes de Odontologia. Foram entregues três instrumentos de avaliação a serem preenchidos, sendo eles o Inventário de Ansiedade de Beck (BAI), Inventário de Depressão de Beck (BDI), e um questionário para registro do perfil de cada estudante. Os testes de Mann Whitney e Kruskal Wallis foram utilizados para comparação dos escores entre os

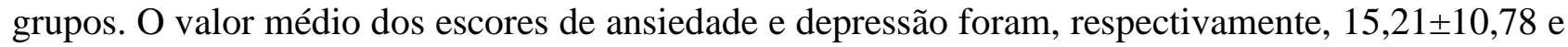
$12,08 \pm 8,38$. Ser calouro, não praticar atividades físicas, ser ateu ou agnóstico, utilizar redes sociais por mais de três horas diárias, vontade de desistir da Odontologia em algum momento do curso, ter procurado ajuda psicológica profissional durante o curso e ter baixa renda foram considerados fatores que resultaram em escores mais elevados de ansiedade e depressão nos universitários. A sintomatologia de depressão e ansiedade foi verificada nos discentes, sendo que características pessoais e acadêmicas podem influenciar em sua saúde mental.
\end{abstract}

Descritores: Depressão. Ansiedade. Estudantes de Odontologia. Qualidade de Vida.

\section{INTRODUÇÃO}

A inserção do jovem na vida acadêmica é uma fase marcada por várias mudanças e adaptações. Do momento em que ingressam nas 
universidades até a conclusão de seus cursos, há uma forte propensão ao desenvolvimento de transtornos psiquiátricos neste grupo populacional. Estima-se que de 15 a $25 \%$ dos estudantes universitários irão apresentar algum tipo de transtorno mental durante sua formação ${ }^{1}$.

Dentre os motivos que tornam os estudantes universitários mais propensos a esse tipo de problema, pode-se citar afastamento do núcleo familiar, em especial dos alunos que acabaram de ingressar nas universidades, intensas cargas horárias, cobrança por parte dos professores das disciplinas, autocobrança relacionada ao não desapontamento dos pais, falta de vocação para o curso escolhido, conflitos sociais de ordem pessoal e inseguranças quanto ao futuro profissional após o fim do curso ${ }^{2}$.

Os acometimentos mais comuns destes distúrbios são a ansiedade e a depressão, sendo a primeira definida como estado de inquietação e tensão sem que haja um motivo específico e, dependendo da sua intensidade e duração, pode gerar sintomas somáticos que causam significativa queda na qualidade de vida de seus portadores ${ }^{3}$. A depressão é um estado mais grave, que leva a um estado extremo de tristeza e isolamento social e, quando não tratada, seu desfecho pode ser o suicídio 4 .

Os níveis de ansiedade e depressão podem variar de acordo com a época do curso em que o estudante se encontra ${ }^{1}$. Cada fase da vida acadêmica gera conflitos e preocupações diferentes. Por exemplo, o estudante calouro passa por uma fase de adaptação e tem que assimilar muitas informações repentinamente; no meio do curso o aluno tem a preocupação de aprimorar-se e ter a certeza de que escolheu a melhor carreira para seu futuro profissional; já no fim do curso, com o término da vida acadêmica, a ansiedade se desenvolve em torno das mudanças de ordem pessoal e profissional que ocorrerão.

Os cursos em si também podem desencadear distintos graus de sintomas de ansiedade e depressão nos discentes, tendo sido constatado, em uma pesquisa, que a Odontologia apresentou os escores mais elevados para ansiedade em comparação com os outros 12 cursos incluídos ${ }^{5}$. A exposição a um ambiente com forte tensão emocional, longas jornadas de trabalho, período de transição do treinamento teórico e pré-clínico para o clínico, alto custo das listas de materiais ${ }^{6}$, e a saída da universidade para o mercado de trabalho são fatores que tornam os estudantes de Odontologia mais predispostos a desenvolverem o transtorno 5 .

Os estudos que exploram a ansiedade e depressão são importantes ferramentas que podem auxiliar no maior entendimento de tais transtornos, que estão se tornando cada vez mais comuns na sociedade atual. Diferentes grupos populacionais podem apresentar níveis distintos da sintomatologia de ansiedade e depressão e, desta forma, os que apresentam maior propensão aos distúrbios, sendo alvo de pesquisas, geram mais evidências para se conhecer qual a proporção do problema e quais os motivos específicos que de fato levam ao seu surgimento.

Desta forma, o objetivo desta pesquisa foi avaliar os estudantes de uma faculdade pública de Odontologia localizada no Brasil, no que diz respeito à ansiedade e depressão, relacionando os escores de cada transtorno com as características pessoais, acadêmicas e socioeconômicas dos discentes.

\section{METODOLOGIA}

Trata-se de um estudo transversal, no qual participaram 423 estudantes de uma faculdade pública brasileira de Odontologia, localizada no estado de São Paulo, dos períodos integral (5 anos de curso) e noturno ( 6 anos de curso). Este tipo de pesquisa visa avaliar a relação entre doenças e/ou outros desfechos em saúde com as variáveis de interesse a serem investigadas, determinando 
também a prevalência da condição nos indivíduos avaliados $^{7}$. A amostra foi composta por todos os alunos regularmente matriculados no ano de 2018, que atenderam aos seguintes critérios de eligibilidade: estar presente no dia da aplicação dos instrumentos de coleta e estar cursando Odontologia nos períodos integral ou noturno. Desta forma, houve a caracterização de amostragem censitária. No período da coleta dos dados, o curso em questão possuía 580 alunos regularmente matriculados, sendo que 400 eram do período integral, com aulas nos períodos matutino e vespertino, e 180 eram do período noturno, com aulas somente à noite. Ressalta-se que o curso possui seu projeto pedagógico alinhado às Diretrizes Curriculares Nacionais.

Para a execução do estudo foram respeitadas e seguidas todas as diretrizes e normas regulamentadoras de pesquisas envolvendo seres humanos da Resolução 466/2012, tendo o mesmo sido aprovado pelo Comitê de Ética em Pesquisa institucional (parecer no. 2.924.654, CAAE 91331918.8.0000.5420). Todos os participantes assinaram o Termo de Consentimento Livre e Esclarecido.

Os questionários foram aplicados por um discente de pós-graduação da instituição de educação superior onde a pesquisa foi realizada, em junho de 2018. Foi preestabelecido um cronograma baseado nos dias e horários disponibilizados por algumas disciplinas que, mediante contato prévio com os professores responsáveis para elucidação das características da pesquisa, gentilmente cederam o tempo necessário para o preenchimento do material durante suas aulas, sendo então a coleta dos dados desenvolvida em salas de aula, laboratórios, ou nas clínicas (antes ou depois do atendimento dos pacientes), em uma única sessão para cada ano de curso.

Como instrumentos de avaliação foram utilizados dois Inventários - o Inventário de Ansiedade de Beck (BAI) e o Inventário de
Depressão de Beck (BDI) - e um questionário para registro do perfil de cada estudante, que requeria informações dos participantes quanto a vários aspectos de sua vida e cotidiano, como idade, prática de atividades físicas, atividades musicoculturais, participação em atividades de iniciação científica ou extensão, renda familiar, reprovações em disciplinas, religião, tempo estimado gasto diariamente em redes sociais, dentre outras variáveis. Para a variável "religião", quatro alternativas poderiam ser assinaladas: 1possui alguma religião, mas não costuma frequentar missas, cultos, reuniões ou encontros; 2 frequenta, de 1 a 2 vezes por mês, missas, cultos, reuniões ou encontros de cunho religioso; 3frequenta, de 3 a 4 vezes por mês, missas, cultos, reuniões ou encontros de cunho religioso; 4- se considera ateu ou agnóstico.

O BAI é um instrumento desenvolvido por Beck et al. $^{8}$ utilizado para se avaliar o rigor dos sintomas de ansiedade. Os indivíduos submetidos a ele respondem 21 afirmativas que refletem somaticamente, cognitivamente e afetivamente os sintomas característicos de ansiedade. O participante deve identificar o quanto tem sido atingido ou incomodado por cada sintoma durante a última semana, incluindo o dia da aplicação. Marca-se com um $X$ na condição que mais representa que sente, numa escala de intensidade com 4 opções: absolutamente não, levemente (não me incomodou muito), moderadamente (foi desagradável, mas pude suportar), e gravemente (dificilmente pude suportar). A avaliação do BAI é somatória, sendo a sintomatologia de ansiedade mais grave quanto mais alto for o escore atingido. A soma dos escores se concentra de 0 a 63 , sendo que cada questão possui resultados de 0 a 3 . A classificação da ansiedade é feita em 4 intervalos, considerando os escores: 0-10, mínimo; 11-19, leve; 20-30, moderado; 31-63, grave.

O BDI é o instrumento para avaliação de depressão, também desenvolvido por Beck et al. ${ }^{9}$, 
que consiste em 21 categorias de sintomas e atitudes, que correspondem a manifestações comportamentais cognitivas afetivas e somáticas da depressão, sendo elas: pessimismo, humor, sentimentos de fracasso, sentimentos de culpa, insatisfação, sentimentos de punição, autodepreciação, autoacusação, desejo de autopunição, irritabilidade, crises de choro, indecisão, isolamento social, inibição do trabalho, crises do sono, fatigabilidade, perda de apetite, perda de peso, perda da libido e preocupação somática. O participante deve identificar o quanto tem sido incomodado por cada sintoma ou situação durante a última semana, incluindo o dia da aplicação. Para a avaliação do BDI, soma-se os escores, classificando-os em 0-11, mínimo; 12-19, leve; 20-35, moderado; e 36-63, grave. Ambos os inventários foram traduzidos e validados para a língua portuguesa, demonstrando características psicométricas satisfatórias ${ }^{10}$.

As análises foram executadas utilizando-se dois programas estatísticos: Epilnfo v. 7.2.2.6 e GraphPad Prism v. 7.00. A normalidade dos dados foi verificada por meio do teste de Shapiro Wilk, que constatou tratar-se de uma distribuição assimétrica. Os testes não-paramétricos de Mann Whitney e Kruskal Wallis, seguidos pelo teste de Dunn para múltiplas comparações, adotando-se o nível de significância de 5\%, foram utililizados para se comparar os escores de ansiedade e depressão.

\section{RESULTADOS}

Os valores médios dos escores de ansiedade e depressão foram, respectivamente, considerando todos os participantes da pesquisa, $15,21 \pm 10,78 \mathrm{e}$ $12,08 \pm 8,38$. Na distribuição percentual dos escores de ansiedade nos intervalos da classificação anteriormente mencionada, foi observado que $41,61 \%$ dos alunos apresentam valores entre 0-10 (mínimo), e os $57,92 \%$ restantes ficaram distribuídos nos intervalos que correspondem às classificações leve, moderado e grave, de acordo com a tabela 1. Quanto aos escores de depressão, $55,08 \%$ dos alunos apresentaram-se no intervalo referente à classificação mínima e 43,03\% distruibuídos nas classificações restantes, verificadas na tabela 2 .

Tabela 1. Distribuição absoluta e percentual dos escores de ansiedade de alunos de uma faculdade de Odontologia brasileira, nos intervalos condizentes com a classificação elaborada por Beck $^{8}$

\begin{tabular}{lcc}
\hline Intervalo & $\mathbf{n}$ & $\mathbf{\%}$ \\
\hline 0-10 (mínimo) & 176 & 41,61 \\
11 a 19 (leve) & 119 & 28,13 \\
20-30 (moderado) & 76 & 17,97 \\
31-63 (grave) & 50 & 11,82 \\
não responderam & 2 & 0,47 \\
\hline Total & $\mathbf{4 2 3}$ & $\mathbf{1 0 0}$ \\
\hline
\end{tabular}

Tabela 2. Distribuição absoluta e percentual dos escores de depressão de alunos de uma faculdade de Odontologia brasileira, nos intervalos condizentes com a classificação elaborada por Beck $^{9}$

\begin{tabular}{lcc}
\hline Intervalo & $\mathbf{n}$ & $\mathbf{\%}$ \\
\hline 0-11 (mínimo) & 233 & 55,08 \\
12 a 19 (leve) & 110 & 26,00 \\
20 a 35 (moderado) & 67 & 15,84 \\
36 a 63 (grave) & 5 & 1,18 \\
não responderam & 8 & 1,89 \\
\hline Total & $\mathbf{4 2 3}$ & $\mathbf{1 0 0}$ \\
\hline
\end{tabular}

Pouco mais de um quarto dos participantes da pesquisa são do sexo masculino $(25,77 \%)$, enquando a grande maioria é composta pelo sexo feminino $(74,23 \%)$. Nas mulheres, os escores médios foram maiores tanto para o BAI $(16,31 \pm 10,93)$ quanto para o BDI $(12,82 \pm 8,28)$, enquanto nos homens os valores foram de $12,03 \pm 9,69$ para ansiedade e 9,91 $\pm 8,33$ para a mensuração da depressão.

As demais variáveis e seus respectivos resultados são apresentados em subcategorias; com estatísticas descritivas que constam das tabelas $3 \mathrm{e}$ 4. 
Tabela 3. Médias, desvios-padrão, valores máximos e mínimos dos escores de ansiedade (BAI) de alunos de uma faculdade de Odontologia brasileira, de acordo com características pessoais e socioeconômicas

\begin{tabular}{|c|c|c|c|c|c|c|}
\hline \multirow{2}{*}{ VARIÁVEIS } & \multirow[b]{2}{*}{$\mathbf{n}$} & \multirow[b]{2}{*}{$\%$} & \multicolumn{4}{|c|}{ ESCORES DE ANSIEDADE } \\
\hline & & & média (desvio padrão) & mínimo & máximo & p-valor \\
\hline \multicolumn{7}{|l|}{ Atividade física } \\
\hline Sim & 234 & 55,3 & $13,33(10,59)$ & 0 & 55 & $<0.0001 *$ \\
\hline Não & 187 & 44,2 & $17,56(10,59)$ & 0 & 57 & \\
\hline Não responderam & 2 & 0,47 & & & & \\
\hline \multicolumn{7}{|l|}{ Atividade musicocultural } \\
\hline Sim & 91 & 21,5 & $16,56(11,58)$ & 0 & 55 & 0,2128 \\
\hline Não & 329 & 44,2 & $14,81(10,55)$ & 0 & 57 & \\
\hline Não responderam & 3 & 0,07 & & & & \\
\hline \multicolumn{7}{|l|}{ Iniciação científica } \\
\hline Sim, sem bolsa & 120 & 28,4 & $15,91(11,16)$ & 0 & 47 & 0,7771 \\
\hline Sim, com bolsa & 78 & 18,4 & $15,26(11,74)$ & 0 & 57 & \\
\hline Não & 222 & 52,5 & $14,84(10,26)$ & 0 & 54 & \\
\hline Não responderam & 3 & 0,71 & & & & \\
\hline \multicolumn{7}{|l|}{ Religião } \\
\hline Possui alguma religião, mas não frequenta & 186 & 44 & $14,7(10,69)$ & 0 & 54 & 0,5548 \\
\hline Frequenta, de 1 a 2 vezes por mês & 100 & 23,6 & $15,39(10,74)$ & 0 & 57 & \\
\hline Frequenta, de 3 a 4 vezes por mês & 79 & 18,7 & $14,87(9,99)$ & 2 & 44 & \\
\hline Se considera ateu ou agnóstico & 54 & 12,8 & $17,37(12,23)$ & 0 & 55 & \\
\hline Não responderam & 4 & 0,95 & & & & \\
\hline \multicolumn{7}{|l|}{ Redes sociais (tempo gasto por dia) } \\
\hline Até 1 hora & 20 & 4,73 & $13,45(13,37)$ & 0 & 57 & $0,0062 *$ \\
\hline Até 1 a 2 horas & 71 & 16,8 & $12,07(9,93)$ & 0 & 37 & \\
\hline De 2 a 3 horas & 116 & 27,4 & $15,42(11,10)$ & 0 & 55 & \\
\hline Mais de 3 horas & 214 & 50,6 & $16,30(10,46)$ & 0 & 55 & \\
\hline Não responderam & 2 & 0,47 & & & & \\
\hline \multicolumn{7}{|l|}{ Odontologia como primeira opção } \\
\hline Sim & 226 & 53,4 & $14,73(10,12)$ & 0 & 55 & 0,602 \\
\hline Não & 192 & 45,4 & $15,81(11,58)$ & 0 & 57 & \\
\hline Não responderam & 5 & 1,18 & & & & \\
\hline \multicolumn{7}{|l|}{ Vontade de desistir do curso } \\
\hline $\operatorname{Sim}$ & 193 & 45,6 & $17,55(11,26)$ & 0 & 57 & $<0.0001 *$ \\
\hline Não & 226 & 53,4 & $13,21(10)$ & 0 & 55 & \\
\hline Não responderam & 4 & 0,95 & & & & \\
\hline \multicolumn{7}{|l|}{ Procura por tratamento psicológico } \\
\hline $\operatorname{Sim}$ & 114 & 27 & $20,15(13,21)$ & 1 & 57 & $<0.0001^{*}$ \\
\hline Não & 308 & 72,8 & $13,65(9,66)$ & 0 & 47 & \\
\hline Não responderam & 1 & 0,24 & & & & \\
\hline \multicolumn{7}{|l|}{ Reprovação em disciplinas } \\
\hline Sim & 91 & 21,5 & $13,35(10,92)$ & 0 & 54 & $0,0259 *$ \\
\hline Não & 329 & 77,8 & $15,69(10,7)$ & 0 & 57 & \\
\hline Não responderam & 3 & 0,71 & & & & \\
\hline \multicolumn{7}{|l|}{ Renda } \\
\hline Até $\mathrm{R} \$ 2500,00$ & 72 & 17 & $18,68(11,83)$ & 1 & 47 & $0,0004 *$ \\
\hline De $R \$ 2501,00$ a 7,500 & 176 & 41,6 & $15,66(11,10)$ & 0 & 57 & \\
\hline Mais que $\mathrm{R} \$ 7500,00$ & 95 & 22,5 & $12,13(9,43)$ & 0 & 48 & \\
\hline Não responderam & 80 & 18,9 & & & & \\
\hline \multicolumn{7}{|l|}{ Ano } \\
\hline $1^{\circ}$ (Integral e Noturno) & 71 & 16,8 & $19,58(10,39)$ & 2 & 48 & $0,0005^{*}$ \\
\hline $2^{\circ}$ (Integral e Noturno) & 97 & 22,9 & $14,88(11,29)$ & 0 & 49 & \\
\hline $3^{\circ}$ (Integral e Noturno) & 108 & 25,5 & $15(9,96)$ & 2 & 54 & \\
\hline $4^{\mathrm{o}}$ (Integral e Noturno) & 52 & 12,3 & $12,88(9,12)$ & 0 & 39 & \\
\hline $5^{\circ}$ (Noturno) & 27 & 6,38 & $15,1(11,9)$ & 0 & 40 & \\
\hline Últimos anos ( $5^{\circ}$ Integral e $6^{\circ}$ Noturno $)$ & 65 & 15,4 & $13,76(11,64)$ & 0 & 57 & \\
\hline Não responderam & 3 & 0,71 & & & & \\
\hline
\end{tabular}


Tabela 4. Médias, desvios-padrão, valores máximos e mínimos dos escores de depressão (BDI) de alunos de uma faculdade de Odontologia brasileira, de acordo com características pessoais e socioeconômicas

\begin{tabular}{|c|c|c|c|c|c|c|}
\hline \multirow{2}{*}{ VARIÁVEIS } & \multirow[b]{2}{*}{$\mathbf{n}$} & \multirow[b]{2}{*}{$\%$} & \multicolumn{4}{|c|}{ ESCORES DE DEPRESSÃO } \\
\hline & & & média (desvio padrão) & mínimo & máximo & p-valor \\
\hline \multicolumn{7}{|l|}{ Atividade física } \\
\hline $\operatorname{Sim}$ & 234 & 55,3 & $10,27(7,32)$ & 0 & 38 & $<0.0001 *$ \\
\hline Não & 187 & 44,2 & $14,31(9,05)$ & 0 & 43 & \\
\hline \multicolumn{7}{|l|}{ Atividade musicocultural } \\
\hline Sim & 91 & 21,5 & $12,73(8,92)$ & 0 & 38 & 0,5202 \\
\hline Não & 329 & 44,2 & $11,9(8,23)$ & 0 & 43 & \\
\hline \multicolumn{7}{|l|}{ Iniciação científica } \\
\hline Sim, sem bolsa & 120 & 28,4 & $12,16(8,48)$ & 0 & 41 & 0,6869 \\
\hline Sim, com bolsa & 78 & 18,4 & $11,19(7,18)$ & 0 & 31 & \\
\hline Não & 222 & 52,5 & $12,4(8,71)$ & 0 & 43 & \\
\hline \multicolumn{7}{|l|}{ Religião } \\
\hline Possui alguma religião, mas não frequenta & 186 & 44 & $11,41(7,89)$ & 0 & 32 & $0,0031 *$ \\
\hline Frequenta, de 1 a 2 vezes por mês & 100 & 23,6 & $11,67(7,99)$ & 0 & 41 & \\
\hline Frequenta, de 3 a 4 vezes por mês & 79 & 18,7 & $11,08(7,63)$ & 0 & 37 & \\
\hline Se considera ateu ou agnóstico & 54 & 12,8 & $16,7(10,26)$ & 0 & 43 & \\
\hline \multicolumn{7}{|l|}{ Redes sociais (tempo gasto por dia) } \\
\hline Até 1 hora & 20 & 4,73 & $9,89(6,84)$ & 0 & 25 & $0,0459 *$ \\
\hline Até 1 a 2 horas & 71 & 16,8 & $10,24(7,67)$ & 0 & 31 & \\
\hline De 2 a 3 horas & 116 & 27,4 & $11,47(7,64)$ & 0 & 32 & \\
\hline Mais de 3 horas & 214 & 50,6 & $13,22(8,96)$ & 0 & 43 & \\
\hline \multicolumn{7}{|l|}{ Odontologia como primeira opção } \\
\hline Sim & 226 & 53,4 & $11,65(8,1)$ & 0 & 40 & 0,3219 \\
\hline Não & 192 & 45,4 & $12,55(8,75)$ & 0 & 43 & \\
\hline \multicolumn{7}{|l|}{ Vontade de desistir do curso } \\
\hline Sim & 193 & 45,6 & $14,66(9,0)$ & 0 & 43 & $<0.0001 *$ \\
\hline Não & 226 & 53,4 & $9,893(7,18)$ & 0 & 32 & \\
\hline \multicolumn{7}{|l|}{ Procura por tratamento psicológico } \\
\hline Sim & 114 & 27 & $15,96(9,96)$ & 0 & 43 & $<0.0001 *$ \\
\hline Não & 308 & 72,8 & $10,64(7,56)$ & 0 & 31 & \\
\hline \multicolumn{7}{|l|}{ Reprovação em disciplinas } \\
\hline Sim & 91 & 21,5 & $12,24(8,41)$ & 0 & 38 & 0,7122 \\
\hline Não & 329 & 77,8 & $12(8,37)$ & 0 & 43 & \\
\hline \multicolumn{7}{|l|}{ Renda } \\
\hline Até $\mathrm{R} \$ 2500,00$ & 72 & 17 & $15,2(9,62)$ & 1 & 43 & $0,0005^{*}$ \\
\hline De $R \$ 2501,00$ a 7,500 & 176 & 41,6 & $11,91(7,84)$ & 0 & 31 & \\
\hline Mais que $\mathrm{R} \$ 7500,00$ & 95 & 22,5 & $9,904(7,96)$ & 0 & 38 & \\
\hline \multicolumn{7}{|l|}{ Ano } \\
\hline $1^{\circ}$ (Integral e Noturno) & 71 & 16,8 & $15,22(9,64)$ & 0 & 41 & \\
\hline $2^{\circ}$ (Integral e Noturno) & 97 & 22,9 & $12,23(9,60)$ & 0 & 43 & $0,0185^{*}$ \\
\hline $3^{\circ}$ (Integral e Noturno) & 108 & 25,5 & $11,92(7,29)$ & 0 & 34 & \\
\hline $4^{\circ}$ (Integral e Noturno) & 52 & 12,3 & $10,23(6,86)$ & 0 & 28 & \\
\hline $5^{\circ}($ Noturno $)$ & 27 & 6,38 & $12,24(8,5)$ & 1 & 32 & \\
\hline Últimos anos ( $5^{\circ}$ Integral e $6^{\circ}$ Noturno $)$ & 65 & 15,4 & $10,82(7,46)$ & 0 & 34 & \\
\hline
\end{tabular}

*diferenças estatisticamente significativas; testes de Mann-Whitney (variáveis dicotômicas) e de Kruskal-Wallis (mais de 2 categorias).

\section{Ano do curso}

Os alunos do primeiro ano apresentaram os escores mais elevados tanto no BAI ( $\mathrm{p}=0,0005)$ quanto no BDI $(\mathrm{p}=0,0185)$, sendo verificadas diferenças estatisticamente significativas entre os grupos.

\section{Atividades físicas}

Com relação à prática de atividades físicas pelos alunos, 55,32\% realizam, ao menos 3 vezes 
na semana, algum tipo de exercício, sendo que $44,21 \%$ assinalaram a opção de que não fazem, e $0,47 \%$ não responderam tal questão.

Nos escores de ansiedade e depressão, houve diferenças estatisticamente significativas entre os dois grupos $(\mathrm{p}=<0,0001$ para ambos os inventários).

\section{Atividades musicoculturais}

Grande parte dos alunos não realiza nenhum tipo de atividade musical ou artística (77,78\%), sendo que apenas $21,51 \%$ responderam positivamente a tal questão.

Nos escores de ansiedade e depressão, não houve diferenças estatisticamente significativas entre os dois grupos $(\mathrm{p}=0,2128$ para o BAI e $\mathrm{p}=0,5202$ para o BDI).

\section{Atividade de Iniciação Científica ou Extensão}

$\mathrm{Na}$ distribuição percentual dos alunos com relação a esta variável, $52,48 \%$ responderam que não realizam, 28,37\% responderam que realizam sem possuir bolsa, e $18,44 \%$ a executam com bolsa. Não foram encontradas diferenças estatisticamente significativas tanto no BAI $(\mathrm{p}=0,7771)$ quanto para o BDI $(\mathrm{p}=0,6869)$.

\section{Religião}

$\mathrm{Na}$ distribuição percentual, 43,97\% dos alunos assinalaram a primeira opção, $23,64 \%$ a segunda, $18,68 \%$ a terceira, e $12,77 \%$ a quarta. Para o BAI não houve diferenças estatisticamente significativas entre os grupos ( $\mathrm{p}=0,5548)$, enquanto para o BDI foi verificado $\mathrm{p}=0,0031$, indicando a presença de diferenças.

\section{Tempo diário médio gasto em redes sociais}

As opções que poderiam ser assinaladas nesta variável eram: até 1 hora; de 1 a 2 horas; de 2 a 3 horas; mais de 3 horas. Mais da metade dos graduandos $(50,59 \%)$ gastam mais que 3 horas de seu dia em redes sociais. Tanto para o BAI $(\mathrm{p}=0,0062)$ quanto para o BDI $(\mathrm{p}=0,0459)$ foram observadas diferenças estatisticamente significativas.

\section{Odontologia como primeira opção de curso}

Consideraram a Odontologia como primeira opção de curso 53,43\% dos graduandos, enquanto 45,39\% assinalaram a esta questão negativamente. Não foram encontradas diferenças estatisticamente significativas tanto para BAI $(\mathrm{p}=0,6020)$ quanto para o BDI $(\mathrm{p}=0,3219)$.

\section{Vontade de desistir realmente da Odontologia em algum momento do curso}

Responderam positivamente a esta questão $45,63 \%$ dos graduandos e, negativamente, $53,43 \%$. Para os dois Inventários houve diferenças estatisticamente significativas, ambas com $\mathrm{p}=<0,0001$.

\section{Tratamento psicológico profissional durante o curso}

Quando indagados se já procuraram tratamento psicológico profissional em algum momento do curso, $26,95 \%$ responderam que sim, enquanto $72,81 \%$ responderam que não, sendo que para os dois inventários foram encontradas diferenças estatisticamente significativas, ambas com $\mathrm{p}=<0,0001$.

\section{Reprovações em disciplina(s)}

Pouco mais de um quinto dos graduandos, $21,51 \%$, possuem reprovação em uma ou mais disciplinas, enquanto $77,78 \%$ responderam que não possuem nenhuma. Para o BAI verificou-se diferenças estatisticamente significativas $(\mathrm{p}=0,0259)$, o que não ocorreu no BDI $(\mathrm{p}=0,7122)$.

\section{Renda}

Com relação aos valores referentes à renda 
familiar mensal, no questionário aplicado aos alunos havia 8 opções para serem assinaladas. Contudo, para realizar-se os testes de comparação, esta variável foi dividida em 3 grupos: 1) até $\mathrm{R} \$ 2500,00 ; 2)$ de $\mathrm{R} \$ 2501,00$ a $\mathrm{R} \$ 7500$; 3) rendas maiores que $\mathrm{R} \$ 7500,00$, sendo que $17,02 \%$ dos alunos estão no primeiro grupo, 41,61\% no segundo, e 22,46\% no terceiro. Para os dois inventários houve diferenças estatisticamente significativas, ambas com $\mathrm{p}=<0,0005$.

\section{DISCUSSÃO}

Nesta pesquisa ficou claramente evidenciado que, com relação ao ano cursado, os alunos ingressantes apresentaram os escores mais elevados tanto de ansiedade quanto de depressão, com diferenças estatisticamente significativas entre os demais anos. A adaptação frente a um novo ambiente, novas rotinas de estudo, novas amizades, afastamento do núcleo familiar, novas responsabilidades, enfrentamento de situaçõesproblema sem o apoio familiar, dentre diversos outros motivos, podem ser responsáveis pela maior sintomatologia de tais distúrbios nos calouros $^{11}$. O sucesso dessa adaptação dos estudantes ao novo ambiente realmente depende de muitos fatores, sendo que alguns deles não estão ligados diretamente ao contexto acadêmico. Apesar disso, a universidade tem um papel fundamental a desempenhar para facilitar esse processo ${ }^{12}$.

Foi constatado no presente estudo que os praticantes de atividades físicas obtiveram escores médios menores tanto no BAI quanto no BDI, com diferenças estatisticamente significativas. Outras pesquisas $^{13,14}$ seguem a mesma tendência deste achado. Um trabalho que objetivou verificar a existência de associação entre o nível de atividade física e a qualidade de vida dos estudantes de Fisioterapia de uma instituição privada de ensino superior comprovou os participantes que possuíam maior nível de qualidade de vida detinham chances maiores de serem ativos ${ }^{13}$. Torna-se alarmente o fato de que os futuros profissinais da área da saúde não atuem como agentes promotores e multiplicadores de hábitos de vida saudáveis, por meio da conscientização de seus pacientes, sendo que talvez nem os próprios tenham esse cuidado em suas vidas ${ }^{14}$. No presente estudo, $44,21 \%$ dos participantes não praticam nenhum tipo de atividade física.

No que concerne às atividades musicoculturais, $77,78 \%$ não realizam nenhum tipo, demonstrando baixo interesse dos participantes. Tanto nos escores do BAI quanto do BDI, não foram encontradas diferenças estatisticamente significativas entre os grupos que praticam e que não praticam tais atividades. Ressalta-se que toda a ação artístico-cultural desempenhada pelo estudante de graduação em programas ou projetos existentes nas Universidades, está incluída no contexto de qualificação e aquisição e desenvolvimento intelectual necessário para a formação de um profissional mais completo ${ }^{15}$.

Com relação a possíveis diferenças nos níveis de ansiedade e depressão entre os graduandos bolsistas, voluntários, e não bolsistas, não foram verificadas diferenças estatisticamente significativas. Seria razoável presumir-se que os bolsistas, por terem que cumprir tarefas extracurriculares, com outras responsabilidades fora da grade de horários da graduação, prazos, envio de relatórios e outros afazeres vinculados à iniciação científica ou atividades de extensão, apresentariam escores mais elevados, o que não foi comprovado. Assim sendo, pode-se dizer que esta variável não afetou a qualidade de vida dos graduandos com relação à ansiedade e depressão. Ademais, a iniciação científica gera impactos positivos na vida pessoal, profissional e acadêmica dos estudantes, 
pois os mesmos têm a oportunidade de desenvolverem habilidades acadêmicas e interpessoais, assim como encontrarem direcionamento profissional ${ }^{16}$.

Para explorar a variável religião, o questionário aplicado aos graduandos não abordou a qual grupo ou segmento religioso eles pertenciam, mas sim qual a frequência de suas práticas religiosas, denotando assim a importância delas em suas rotinas. A maior parte dos participantes, 43,97\%, diz possuir alguma religião, mas não costumam frequentar missas, cultos ou encontros. Este mesmo grupo apresentou, tanto no BAI quanto no BDI, os menores escores médios. Em contrapartida, os que se definiram como ateus e agnósticos $(12,77 \%)$ obtiveram os maiores valores médios para ansiedade e depressão, sendo que para esta última, foram observadas diferenças estatisticamente significativas, indicando que este grupo sofre mais com tal transtorno. Tal achado está em consonância com estudos semelhantes, que mostraram que uma das estratégias de enfrentamento de situações que geram estresse ou desencadeiam a sintomatologia de ansiedade é a busca de apoio na religião ${ }^{17} \mathrm{e}$, mais ainda, a espiritualidade foi considerada como uma forte ferramenta de confronto a situações adversas por meio do coping religioso-espiritual, que se trata da maneira como os indivíduos utilizam a fé para lidar com o estresse, tendo se associado com melhores índices de qualidade de vida e saúde física e mental ${ }^{17,18}$.

O uso das redes sociais, que teve sua ascensão a partir do século XXI, é um fenômeno cultural humano relativamente recente, $\mathrm{e}$ as consequências desta nova prática na rotina dos indivíduos têm sido alvos de estudos contemporâneos, principalmente buscando associar o uso excessivo das mídias a sintomas patológicos comportamentais ${ }^{19}$. Um estudo observou que acadêmicos de Medicina apresentaram o espectro dos aspectos nocivos que o uso excessivo da internet pode configurar, representado em sintomatologia ansiosa e depressiva, prejudicando o rendimento acadêmico ${ }^{20}$. Inúmeros efeitos deletérios podem ser desencadeados pelo uso descontrolado das redes sociais, como alterações na qualidade do sono, na nutrição e na atividade física, queda no desempenho acadêmico ou profissional e prejuízo nas relações interpessoais, transtornos de humor, transtorno de déficit de atenção e hiperatividade, transtorno de uso de substâncias, baixa autoestima, ansiedade, ansiedade social, solidão, comportamento agressivo e hostilidade, comportamento compulsivo, impulsividade, maiores taxas de transtornos de personalidade, prejuízos na saúde mental de forma geral e, em casos mais graves, suicídio ${ }^{21-23}$.

$\mathrm{O}$ presente estudo constatou que mais de metade dos estudantes de Odontologia gastam 3 horas ou mais nas redes sociais diariamente, e que tanto nos escores de ansiedade quanto depressão, este grupo apresentou os maiores valores médios, tendo ainda sido verificadas diferenças estatisticamente significativas. A questão a ser elucidada agora é se o mau uso da internet e tempo excessivo gasto em redes sociais podem gerar a sintomatologia de ansiedade e depressão, ou se o uso delas representa um mecanismo de compensação para aqueles que já possuem propensão a esses distúrbios ${ }^{24,25}$.

Quanto à escolha do curso, 45,39\% dos graduandos assinalaram que a Odontologia não era a primeira opção. Destes, 54,69\% tinham como primeira escolha o curso de Medicina. Com a possível frustração que tal situação pudesse causar, seria plausível considerar que níveis maiores de depressão e ansiedade fossem observados neste grupo e, apesar de serem constatados de fato valores médios maiores do BAI e BDI no grupo que não tinha a Odontologia 
como primeira opção, não foram observadas diferenças estatisticamente significativas. Por outro lado, quando indagados quanto à vontade de desistir da Odontologia em algum momento do curso, houve diferenças estatisticamente significativas entre os grupos, sendo que os que assinalaram "Sim" apresentaram os maiores escores de depressão e ansiedade. Desta forma, pode-se pressupor que os problemas gerados no decorrer do curso têm maior impacto do que a dúvida inicial com relação a sua escolha, no que diz respeito ao desencadeamento dos sintomas de depressão e ansiedade, ressaltando-se que outras variáveis não tratadas neste estudo também podem influenciar diretamente no surgimento dos sintomas.

Aproximadamente um quarto dos participantes desta pesquisa $(26,95 \%)$ procurou ajuda psicológica em profissionais especializados durante o curso. Tanto para os escores de depressão quanto para os de ansiedade, o grupo que respondeu positivamente a esta questão apresentou os maiores valores médios, com diferenças estatisticamente significativas. Pode-se então presumir que os graduandos que possuíam maiores sintomatologias das condições estudadas procuraram ajuda psicológica para tratar do problema, mas não necessariamente conseguiram atenuar os sintomas, o que resultaria em valores mais baixos dos escores. Grande parte dos indivíduos com algum tipo de transtorno psiquiátrico não procura tratamento em profissionais especializados ${ }^{26}$. O perfil da maioria dos universitários que procura tratamento psicológico em programas de atendimento dentro das próprias universidades segue o padrão de se tratarem de alunos ingressantes, com idade entre 19 e 22 anos, do gênero feminino, que não exerce atividade profissional remunerada, e que apresentam "dificuldades psicológicas moderadas" 26 , e que estão relacionadas tanto com situações internas (sofrimento mental) quanto de situações ambientais e interpessoais ${ }^{27}$. Ressalta-se que a faculdade onde foi realizado o presente estudo oferece acompanhamento psicológico aos graduandos gratuitamente.

A reprovação em disciplinas pode resultar em anos a mais de curso, o que geraria em atraso na conclusão do mesmo. Neste estudo foi constatado que $21,51 \%$ dos alunos possuem reprovação em uma ou mais disciplinas. Curiosamente, o grupo que respondeu não possuir nenhuma reprovação apresentou os maiores valores médios do BAI, com diferenças estatisticamente significativas. Pode-se entender que, para manter as boas notas e o bom rendimento acadêmico, os estudantes se cobrariam mais nos estudos, resultando em maior estresse, o que poderia gerar os sintomas de ansiedade exacerbados. Um estudo semelhante constatou que apesar de a maioria dos participantes de seu estudo (74\%) terem apresentado sinais de estresse, o desempenho acadêmico dos mesmos permaneceu acima da média $^{28}$. Para o BDI, os valores médios entre os grupos foram semelhantes, sem diferenças significativas.

Com relação às rendas familiares dos estudantes, tanto para ansiedade quanto para depressão, os maiores e menores escores médios foram verificados, respectivamente, no grupo cuja renda atingia o valor máximo de $\mathrm{R} \$ 2500,00$, e no grupo cuja renda ultrapassava $R \$ 7500,00$, sendo ainda verificadas diferenças estatisticamente significativas. $\mathrm{Ou}$ seja, as possíveis dificuldades econômicas enfrentadas pela família do graduando em mantê-lo em um curso considerado caro, principalmente devido às compras de materiais e instrumentais, são potenciais causadores da queda de sua qualidade de vida, refletidos nas sintomatologias das desordens psicológicas tratadas neste estudo ${ }^{6}$. 
Salienta-se que, apesar de o BAI e o BDI serem instrumentos comprovadamente eficazes em pesquisas com esta temática, a complexidade da depressão e da ansiedade enquanto condições psicológicas podem requerir, dependendo do objetivo específico do pesquisador, outras formas de dignóstico com outras abordagens metodológicas, e esta situação pode configurar uma limitação deste estudo. Sendo assim, o desenvolvimento de mais trabalhos semelhantes deve ser estimulado, principalmente em populações onde estes acometimentos são mais prevalentes, resultando em maiores evidências científicas sobre a extensão deste problema, que vem se tornando cada vez mais presente na população.

É importante se destacar que os Inventários utilizados neste trabalho fornecem indícios de ansiedade e depressão, sendo que o diagnóstico e tratamentos adequados necessitam de orientação e supervisão dos profissionais da área.

Há um consenso crescente de que os universitários são mais acometidos por desordens psicológicas ${ }^{1,2,5} \mathrm{e}$, desta maneira, estudos que explorem a saúde mental deste grupo populacional tornam-se necessários, principalmente para se conhecer a extensão do problema e quais os fatores desencadeiam tais condições. Em especial, a Odontologia possui características específicas que podem alavancar ainda mais tais sintomas ${ }^{5,6}$.

\section{CONCLUSÃO}

Ser calouro, não praticar atividades físicas, ser ateu ou agnóstico, utilizar redes sociais por mais de três horas diárias, pretensão de desistência em algum momento do curso, ter procurado ajuda psicológica em profissionais durante o curso, e ter baixa renda, foram considerados fatores que resultaram escores mais elevados de ansiedade e depressão nos universitários.

\section{ABSTRACT \\ Factors associated with the development of anxiety and depression in dental students}

The aim of this study was to evaluate the students of a public dental school located in Brazil, regarding anxiety and depression, relating the scores of each disorder with the students' personal, academic, and socioeconomic characteristics. This is a cross-sectional study in which 423 dental students participated. Three assessment instruments were submitted to be completed, namely the Beck Anxiety Inventory (BAI), Beck Depression Inventory (BDI), and a questionnaire to record the profile of each student. Mann Whitney and Kruskal Wallis tests were used to compare scores between groups. The average value of anxiety and depression scores were, respectively, $15.21 \pm 10.78$ and 12.08 \pm 8.38. Being freshman, not practicing physical activities, being atheist or agnostic, using social networks for more than three hours a day, willingness to give up dentistry at some point of the course, having sought psychological help from professionals during the course, and having low income, were factors that resulted in higher scores for anxiety and depression in college students. The symptoms of depression and anxiety were verified in the students, and personal and academic characteristics can influence their mental health.

Descriptors: Depression. Anxiety. Students, Dental. Quality of Life.

\section{REFERÊNCIAS}

1. Vasconcelos TC, Dias BRT, Andrade LR, Melo GF, Barbosa L, Souza E. Prevalência de sintomas de ansiedade e depressão em estudantes de medicina. Rev Bras Educ Med. 2015; 39(1):135-42.

2. Catunda MAP, Ruiz VM. Qualidade de vida de universitários. Pensam Plur. 2008; 2(1):22-31.

3. Andrade JV, Pereira LP, Vieira PA, Silva JVS, Silva AM, Bonisson $\mathrm{M}$, et al. Ansiedade: um dos problemas do século 
XXI. Rev Saúde ReAGES. 2019; 2(4):34-9.

4. Barros MBA, Lima MG, Azevedo RCS, Medina LBP, Lopes CS, Menezes PR, et al. Depressão e comportamentos de saúde em adultos brasileiros: PNS 2013. Rev Saúde Pública. 2017; 51(suppl 1):1.

5. Victoria MS, Bravo A, Felix AK, Neves BG, Rodrigues CB, Ribeiro CCP, et al. Níveis de ansiedade e depressão em graduandos da Universidade do Estado do Rio de Janeiro (UERJ). Encontro Rev Psicol. 2013; 16(25):163-75.

6. Madhan B, Barik AK, Patil R, Gayathri H, Reddy MSR. Sense of humor and its association with psychological disturbances among dental students in India. J Dent Educ. 2013; 77(10):1338-44.

7. Porta AN. a dictionary of epidemiology. 6. ed. New York: Oxford University Press, 2014.

8. Beck AT, Brown G, Epstein N, Stter RA. An inventory for measuring clinical anxiety: psychometric properties. J Consult Clin Psychol. 1988; 56(6):893-97.

9. Beck AT, Ward CH, Meldenson M, Mock J, Erbauch G. An inventory for measuring depression. Arch Gen Psychiatry. 1961;4:561-71.

10. Cunha JA. Manual da versão em português das Escalas Beck. São Paulo: Casa do Psicólogo, 2001.

11. Santos BRM, Gonzales PS, Carrer FCA, Araújo ME. Perfil e expectativas dos ingressantes da Faculdade de Odontologia da USP: uma visão integrada com as diretrizes curriculares nacionais e o sistema único de saúde. Rev ABENO. 2015; 15(1):28-37.

12. Teixeira MAP, Dias ACG; Wottrich SH, Oliveira AM. Adaptação à universidade em jovens calouros. Psicol Esc Educ. 2008; 12(1):185-202.
13. Costa PHV, Silva FS, Machado CJ. Nível de atividade física e qualidade de vida dos estudantes de fisioterapia de uma instituição privada de ensino superior. Rev Interdiscip Ciênc Méd. 2018; 1(2):46-53.

14. Silva DAS. Indicadores do estilo de vida e autoavaliação negativa de saúde em universitários de uma instituição pública do nordeste do Brasil. Rev Bras Ativ Fis Saúde. 2012; 17(4):263-69.

15. Mira P, Martins J, Zambonini G, Oliveira SA, Aires CP. Contribuição de monitores de graduação nas atividades do coral da USP Ribeirão Preto: o trabalho nos bastidores. Rev Grad USP. 2018; 3(1):83-90.

16. Pinto NLS, Fernandes LM, Silva FF. Para além da formação acadêmica: as contribuições da iniciação científica para o desenvolvimento pessoal e profissional de estudantes da área de administração. RAEP. 2016; 17(2):301-25.

17. Esperandio MRG, Escudero FT, Fanini L, Macedo EPN. Envelhecimento e espiritualidade: o papel do coping espiritual/religioso em pessoas idosas hospitalizadas. Interaçao psicol. 2019;23(2):268-80.

18. Seleghim MR, Mombelli MA, Oliveira MLF, Waidman MAP, Marcon SS. Sintomas de estresse em trabalhadoras de enfermagem de uma unidade de Pronto Socorro. Rev Gaúcha Enferm. 2012; 33(3):165-73.

19. Moromizato MS, Ferreira DBB, Souza LSM, Leite RF, Macedo FN, Pimentel D. O uso de internet e redes sociais e a relação com indícios de ansiedade e depressão em estudantes de medicina. Rev Bras Educ Med. 2017; 41(4):497-504.

20. Mazhari S. The prevalence of problematic internet use and the related factors in medical students, Kerman, Iran. Addict 
Health. 2012; 4(3-4):87-94.

21. Christakis DA. Internet addiction: a $21 \mathrm{st}$ century epidemic? BMC Med. 2010; 8:61.

22. Ko CH, Yen JY, Yen CF, Chen CS, Chen CC. The association between internet addiction and psychiatric disorder: A review of the literature. Eur Psychiatry. 2012; 27(1):1-8.

23. Menezes PP. O virtual, o homem e a Psicanálise. Rev Reverie. 2012; 5(1):100-9.

24. Dong G, Lu Q, Zhou H, Zhao X. Precursor or sequela: pathological disorders in people with internet addiction disorder. PLoS One. 2011; 6(2):e14703.

25. Guimarães AMV, Silva Neto AC, Vilar ATS, Almeida BGC, Albuquerque CMF, Fermoseli AFO. Transtornos de ansiedade: um estudo de prevalência sobre as fobias específicas e a importância da ajuda psicológica. Ciênc Biol Saúde. 2015; 3(1):115-28.
26. Neves MCC, Dalgalarrondo P. Transtornos mentais auto-referidos em estudantes universitários. J Bras Psiquiatr. 2007; 56(4):237-44.

27. McLafferty M, Lapsley CR, Ennis E, Armour C, Murphy S, Bunting BP, et al. Mental health, behavioural problems and treatment seeking among students commencing university in Northern Ireland. PLoS One. 2017; 12(12):e0188785

28. Mondardo AH, Pedon EA. Estresse e desempenho acadêmico em estudantes universitários. Rev Ciênc Hum. 2005; 6(6):159-80

\section{Correspondência para:}

Clea Adas Saliba Garbin email:c.garbin@foa.unesp.br

Rua José Bonifácio, 1193

16015-050 Araçatuba/SP 